\title{
Die Tiefen der Erdbebenherde in Dentschland auf Grund makroseismischer Berechnungen
}

\author{
W. SPONIIEUER
}

\section{Zusammenfassung}

Über die Tiefe der Erabebenherde in Deutschland wabrisher nur wenig bekaunt. Isclignlich fur das Gebiet der Schwäbischen Alb konnten lank dem Stationsmetz res Wüuttembergischen Errberbendienstes die Heraltiefen von neueren Erabeben mikroseismisch bestimmt werelen. Fur alle anderen Herregebiete Deutschlands lagen bisher werler mikroseismisch noch makroseismisch

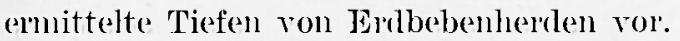

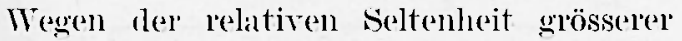
Erolbeben innerhalb Deutschlands und ales Fohlens gexigneter fur Heriticenbestimmungen auswertbaler Registriemengen wurden nachfolgend nur Ileritiefen derjenigen Errbeben makroseismisell ementtelt, von denen eine Tsoseistenkartegezeichnet werden kominte.

IIt Hilfe einer nenartigen Methorle (i) ist es möglich, nit geringem /eitanfwand eine grössere /jahl von Erdberben zu berabeiten und Eluebuisse zu erzielen, die mit mikroseismisch errecluneten gut übereinstimmen.

Wie ren meisten bisher bekannten Verfaluen liegt anch rlen hier benutzten die Gleichumg von Köressligethy (5) zugrumile.

$$
I_{o}-I=3 \log \frac{r}{h}+3 \alpha I I(r-h),
$$

orler, rla nach Abb. 1

$$
\begin{gathered}
r^{2}=h^{2}+s^{2}, \\
I-I_{o}=3 \log \frac{1}{h} \mid h+s^{2}+ \\
+3 a \mathrm{M}\left(\mid h^{2}+s^{2}-h\right) .
\end{gathered}
$$

Hierbei ist

$I_{o}=$ Intensität ins Epizentrum [Grad]

$I=$ Intensität in der Entferomung $s$ vom Epizentrum

$s=$ mittlere Entlernung der Isoseiste I vom Epizentrum (Isoseistemrallius)[km]

$h=$ Hereltiefe $[\mathrm{km} n]$

$\alpha=$ Absor'ptionskoeffizient $[\mathrm{km}]^{-1}$

$M=\log e=0,4343$.

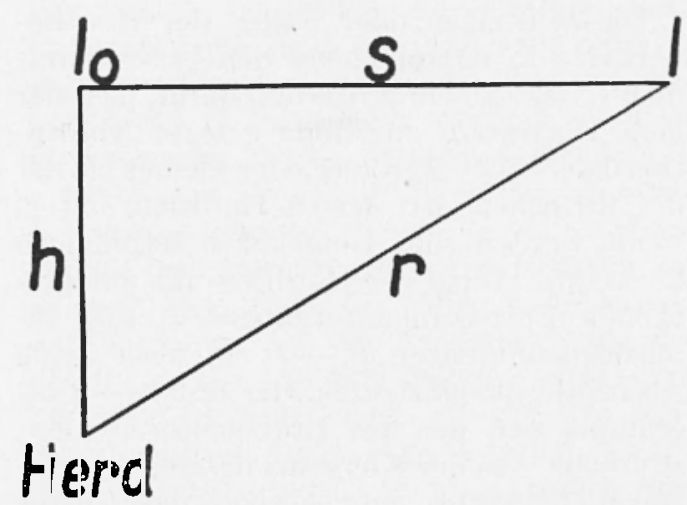

Abb. 1 .

In einem Diagramm nach Gleichung [2] kann nun fur einen festen Wert des . $\mathrm{b}$ sorptionskoeffizienten a die Herdtiefe $h$ in Abhangigkeit von der Epizentralentfernung dirgestellt werden (Abb. 2)

Stimmt zufällig der Wert von a. der diesem Diagramm zugrunde liegt, mit dem eines Erdbebens überein, so muss sich zu jerlem Isoseistenradlius s und der zugehörigen Intensitätsdifferenz $I_{0}-\mathrm{I}_{n}$ ein konstanter Wert der Herdtiefe $h$ ablesen lassen. In allen anderen Fällen, in denen der feste 
Wert $a$ des Jiagramms ron dem des Erdbebens rerschieden ist, werden die abgelesenen Herdtiefenwerte, die den Entfernungen $s_{n}$ und den Intensitätsdifferenzen $I_{o}-\mathrm{I}_{n}$ entsprechen, mit grosser werdender Epizentralentfermung entweder ansteigen oder fallen, wie es in $\Delta b b .2$ fur $\alpha=0,02$ und $a=0,0+$ gezeigt wircl.

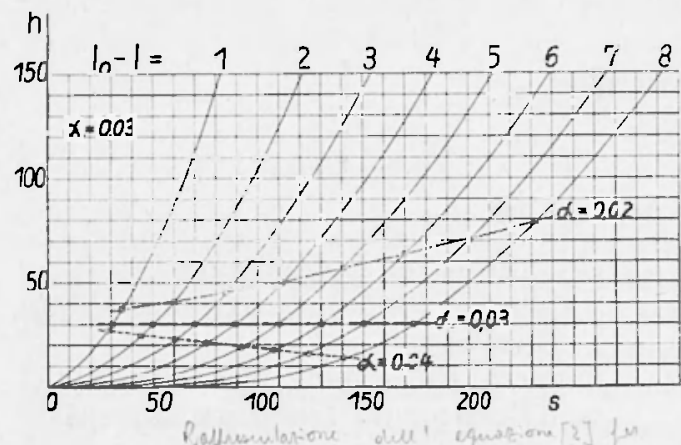

Abb. 2. - Darstellung von Gleichung [2] für festes a umr ganzzahlige Intensitätsdifterenzen $I_{o}-I$.

Dioses Steigen oder Fallen der Herdtiefenwerte $h_{n}$ entsprechend den Isoseistenradien $s_{n}$ ist also ein Kriterium dafür, dass der dem Diagramm zugrunde gelegte $A$ bsorptionskoeffizient a grosser odler kleiner als der des Erdbebens ist, dessen Herdtiefe untersucht werden soll. Ungekehrt zeigen also konstante Werte der Herdtiefe für entsprechende Epizentralentfernungen $s_{n}$ und intensitätsdifferenzen $I_{o}-I_{n}$ an, dass beide Absorptionskoeffizienten, der feste des Diagramms und der des Erdbebens, übereinstimmen. Aus der Konstanz des abgelesenen Herdtiefenwertes geht hervor, dass dieser der gesuchte richtige Wert ist. Hierbeiwird wie bei allen anderen makroseismischen Herrdtiefenbestimmungsverfahren stillschweigend vorausgesetzt, dass der Absorptionskoeffizient eines Erdbebens für verschiedene Herdentfernungen sich nicht ändert. Man konnte nun mit Hilfe der vorstehenden Betrachtung eine Nethode zur Bestimmung der Herdtiefe gewinnen. Man benötigt dazu eine Anzall von Diagrammen nach $\Lambda \mathrm{bb} .2 \mathrm{mit}$ himreichend abgestuften festen Werten des Absorptionskoeffizienten a. Durch Probieren, also in Näherungsverfahren, wird nun dasjenige Diagramm herausgesucht, das einen konstanten, d. h. rich- tigen Wert der Herdtiefe für jeden gegebenen Isoseistenrarlius $s$ und Intensitätsdifferenz $I_{o}-I$ ablesen lasst. Dieser Methode stehen lediglich in Wege, dass eimmal eine grosse Zahl derartiger Diagramme notwendig ist, zum anderen, dass die Herstellung solcher Diagramme infolge der Transzendenz der Gleichung [2] sehr mühevoll und zeitraubend ist. Um diese Schwierigkeit $\mathrm{zu}$ umgehen, wurde von Ullmann $\left({ }^{*}\right)$ in Gleichung [1] der Absorptionskoeffizient $\alpha$ eliminiert, wodurch sich die Gleichung in eine bequeme Form bringen lässt. Er stellt folgende Definitionsgleichungen auf.

oder

$$
\eta=a h, \varrho=a r, \sigma=\alpha s
$$

$$
\frac{\eta}{h}=\frac{\varrho}{r}=\frac{\sigma}{s}=\alpha .
$$

Die Zuordnung dieser Grössen geht aus Abb. 3 hervor, und man kamn nun [1] wie folgt schreiben.

$$
\frac{I_{0}-I}{3}=\log \frac{\rho}{\eta}+\mathrm{M}(\varrho-\eta) .
$$

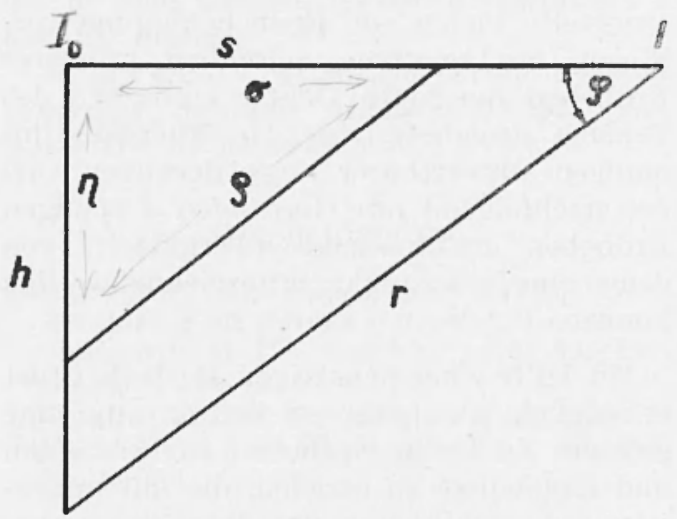

Abb. 3. - Zur Herleitung von Formel [10].

Nach rer $\mathrm{Abb} .3$ ist

und daher

$$
\frac{g}{\eta}-\frac{1}{\sin \varphi}
$$

$$
\varrho-\eta=\varrho(1-\sin \varphi) .
$$

Nun wird

$$
\frac{I_{0}-I}{3}=-\log \sin \varphi+M \varrho(1-\sin \varphi)
$$

(*) Dr. W. Ullmann, Inst. für Bodendynamik und Erdbebenforschung, Jena. 
daraus

$\varrho=\left(\frac{I_{n}-I}{3}+\log \sin \varphi\right) \frac{1}{M} \frac{1}{1-\sin \varphi} \cdot[6]$

Setzt man

$$
\begin{gathered}
c(\varphi)=\frac{1}{M} \frac{1}{1-\sin \varphi}, \\
-c(\varphi) \log \sin \varphi=f(\varphi) . \\
\gamma=\frac{I_{o}-I}{3},
\end{gathered}
$$

damn ergibt sich fur [6]

$$
\varrho=\gamma c(\varphi)-f(\varphi) \text {. }
$$

$o$ ist nur definiert für

$$
\gamma=\log \sin \varphi>0
$$

odler

folglich

$$
\ddot{\gamma}+\ln \sin \varphi>0
$$

$$
\sin m>e^{-\frac{\gamma}{M}}
$$

Mit Hilfe von Gleichung [10] lassen sich für alle Intensitätsdifferenzen $I_{0}-I_{n}$, die zweckmässig um halbe oder viertel Grade abgestuft werden, Kurven berechnen, da die Ausdrücke [7, 8, 9] leicht tabellierbar sind und muhsame Näherungsberechnungen fortfallen. Entweder liann man mit dem Winkel $\varphi$ und der Grösse $\varrho$ für jedes $\gamma$ eine Kurve zeichnen, oder man benutzt dazu die Grössen $\sigma$ und $\eta$, die sich aus

und

$$
\eta=0 \sin \varphi
$$

ergeben.

$$
\sigma^{2}=Q^{2}+\eta^{2}
$$

Die Bestimmung der Herdtiefe eines Erdbebens mittels des $\sigma, \eta$-Diagramms geht nun folgendermassen vor sich: Auf Grund der Isoseistenkarte sind die mittleren Isoseistenabstände rom Epizentrum $s_{n}$ für die entsprechenden Intensitätsdifferenzen $I_{n}-I_{n}$ sowie die Intensität im Epizentrum $I_{o}$ gegreben. Unter Innahme eines geschatzten Trertes für die Absorption a erliält man durch einfache Multiplikation mit den Isoseistemradien $s_{n}$ die Grössen $\sigma_{n}$.
Num entnimmt man dem Diagramm zu jedem $\sigma$ bei der zugehörigen Intensitätsdifferenz $I_{o}-I$ das entsprechende $\eta$. Steigen oder fallen die $\eta$-WVerte, so wird der Vorgang mit einem kleineren bzw. grösseren Wert von a solange, wiederholt, bis die $\eta$ Terte konstant bleiben. Aus $\eta$ erhält man durch Division mit a die gesuchte Herdtiefe h. Selbstrerständlich muss in Katuf genommen werden, dass infolge der niemals idealen Verteilung der Isoseisten die $\eta$ Werte streuen. Jedoch lässt sich fast immer erkennen, ob sie eine fallende oder steigende Tendenz haben. Stärker streuende Werte zeigen an, dass die zugehörigen Isoseistenradien zu stark fehlerhaft und daher nichit geeignet für eine Herdtiefenbestimmung sind.

Da bei diesem Verfalnen die Absorption a zunächst geschätzt werden muss, sind meist mehrere Näherungsschritte nötig, um den richtigen, d. h. konstanten Wert der IIerdtiefe zu ermitteln. Ferner geht aus Gleichung [1] hervor, dass die Epizentralintensität $l_{o}$ möglichst genau bekannt sein muss, um sichere Erocbnisse zu erhalten. Man kamn aber die Zahl der Näherungsschritte stark herabsetzen, wenn der Absorptionskoeffizient nicht belicbigr gewählt, sondern in geeigneter Weise abgeschätzt wird.

Stellt man Gleichung [2] so dar, dass die Intensitätsdifferenz $I_{n}-I$ in Abhängigkeit vom Isoseistenradius s gebracht wird, so stellt diese Funktion (Abb. 4) die Intensitätsabnahme mit der Entfernung für die Gleichung von Kövesligethy dar. Aber auch für jedes Erdbeben lässt sich mit den makroseismischen Daten eine derartige Kurve zeichnen, wenn man $I$ in Abhängigkeit von $s$ aufträgt. Tergleicht man eine derartige Intensitätsabnahmekurve mit den theoretischen Intensitätsabnahmekurven etwa durch Übereinanderlegen der auf transparentem Papier gezeichneten Diagramme, so lässt sich einmal der Wert des Absorptionskoeffizienten $a$ mit genngender Genauigkeit abschätzen, zum anderen zeigt die zn der Bebenkurve passende theoretische $\mathrm{Ab}$ nahmekurve den extrapolierten Wert der Intensität $I_{o}$ limreichend genau an. Ausserdem ist es in vielen Fallen möglich, auch den ungefähren Wert der Herdiefe $h$ aus dem Diagramm zu entnehmen, so dass Irrtümer bei der Herdtiefenberechnung ver- 
mieden werden konnen. Mit Hilfe des abgeschätzten Wertes von $a$ kann die Ermittlung der Herdtiefe nach dem vorher beschriebenen Verfahren vorgenommen werden. tische mit $\alpha=0,001$. Legt man die beiden Kurven so übereinander, dass die Epizentralintensität ron 6 Grad mit dem Nullpunkt des Koordinatensystems der theoretischen Kurve zusammenfällt, so liegen die

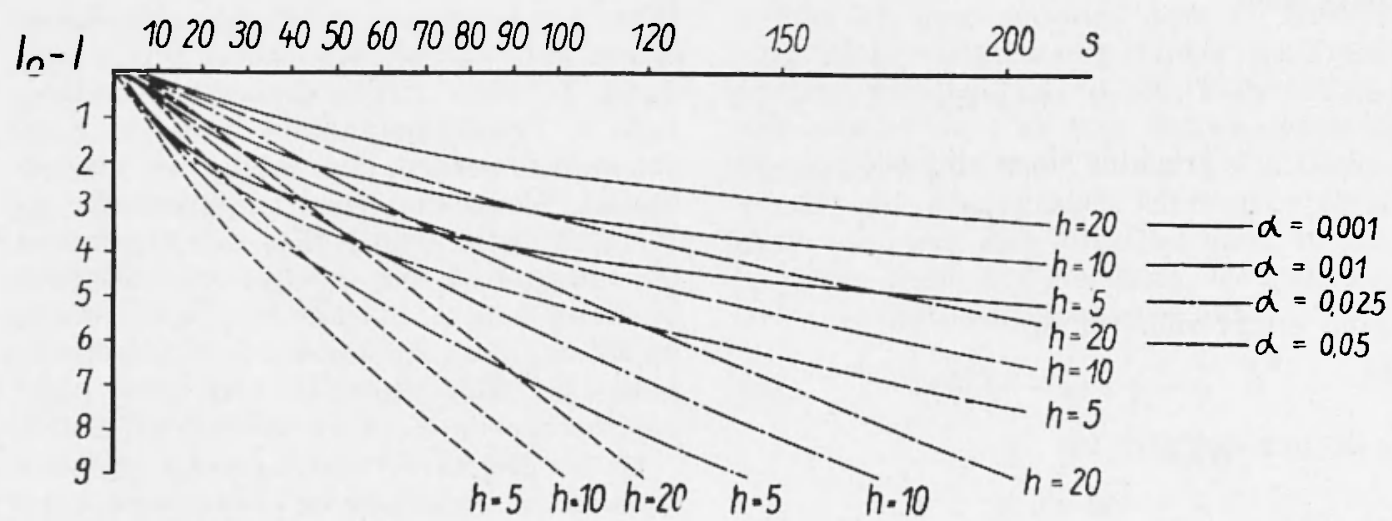

Abb. 4. - Abhängiqkeit der Intensitütsdifferenz $I_{o} I$ von der Entfermung $s$ für verschiedene ILerdtiefen $h$ und Absorptionskoeffizienten $a$ nach Gleichung [2].

Das Verfahren moge an einem Biespiel erlautert werden. (Erdboben an der Hornisgrinde vom 30.12 .1935$)$. beobachteten Epizentralentfernungen ungefähr auf der theoretischen Kurve für $h=$ $30 \mathrm{~km}$. (Abb. 5).

\section{$s[\mathrm{~km}]$}

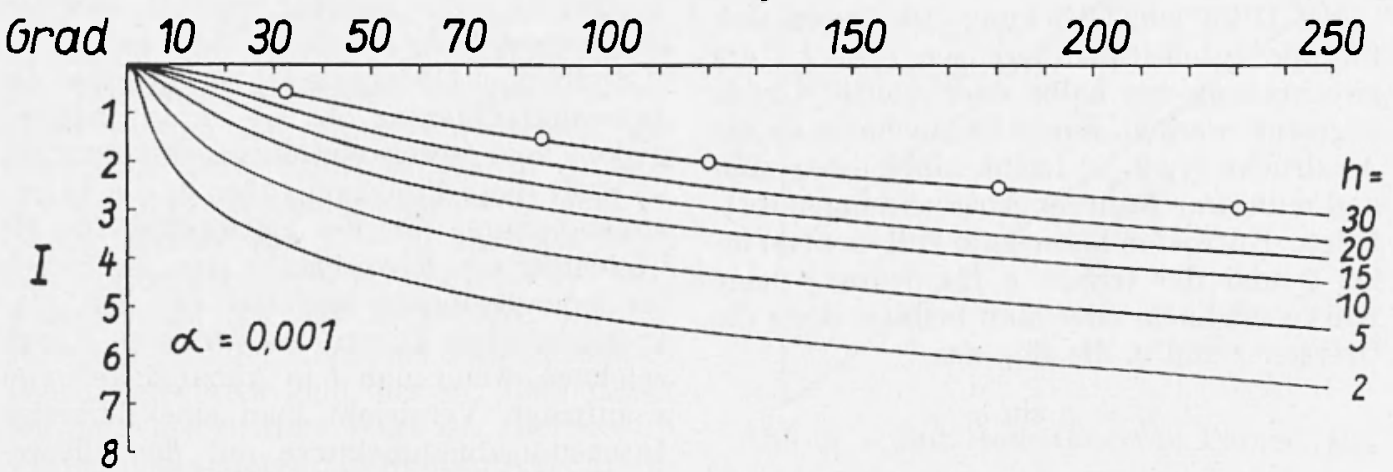

Abb. 5. - Abschätzung von $a$ und $h$ durch Anpassen der makroseismischen Daten an eine Kurvensehar nach Gleichung [?].

Der Isoseistenkarte von Hiller (') wurden folgende mittlere Isoseistenradien entnommen:

$$
\begin{array}{lllcccc}
I_{n} & 5,5 & 4,5 & 4 & 3, \tilde{5} & 3 & \text { Grand } \\
\hline s_{n} & 32 & 85 & 120 & 180 & 230 & \mathrm{~km}
\end{array}
$$

Trägt man $s$ in Abhängigkeit von $I$ auf, so erhält man für diese Intensitätsabnahmekurve die beste Annäherung an die theori-
Tach den makroseismischen Beobachtungen ist allerdings $I_{o}$ höher als 6 Grad, jedoch niedriger als 6,5 Grad gewesen, so dass $I_{0}=6,25$ gesetzt wurde. Daher muss die Herdtiefe geringer als $30 \mathrm{~km}$ sein, wie sich auch aus dem Kurvenvergleich ergibt, wenn man die Ordinate ron 6,25 auf den Ursprung der theoretischen Kurve legt. Mit $\alpha=0,001$ erhält man, da $\sigma=s a$, für die 
entsprechenden In t e ns it ä ts $\mathrm{d}$ i ff e re $n \mathrm{z}$ e n $I_{0}-I_{n}$.

$$
\begin{array}{ccccccc}
I_{0}-I_{n} & 0,75 & 1,25 & 1,75 & 2,25 & 2,75 & 3,25 \\
\hline \sigma_{n} & 0,032 & 0,05 & 0,085 & 0,120 & 0,180 & 0,230
\end{array}
$$

Der' Vorteil der anfgezeigten Methode liegt ror allem darin, dass zu jedem Wert der mittleren Isoseistenentfermung ein Herdtiefenwert betmmtwird. Strenen die erlaltenen Werte der Herdtiefe inmerhalb eines

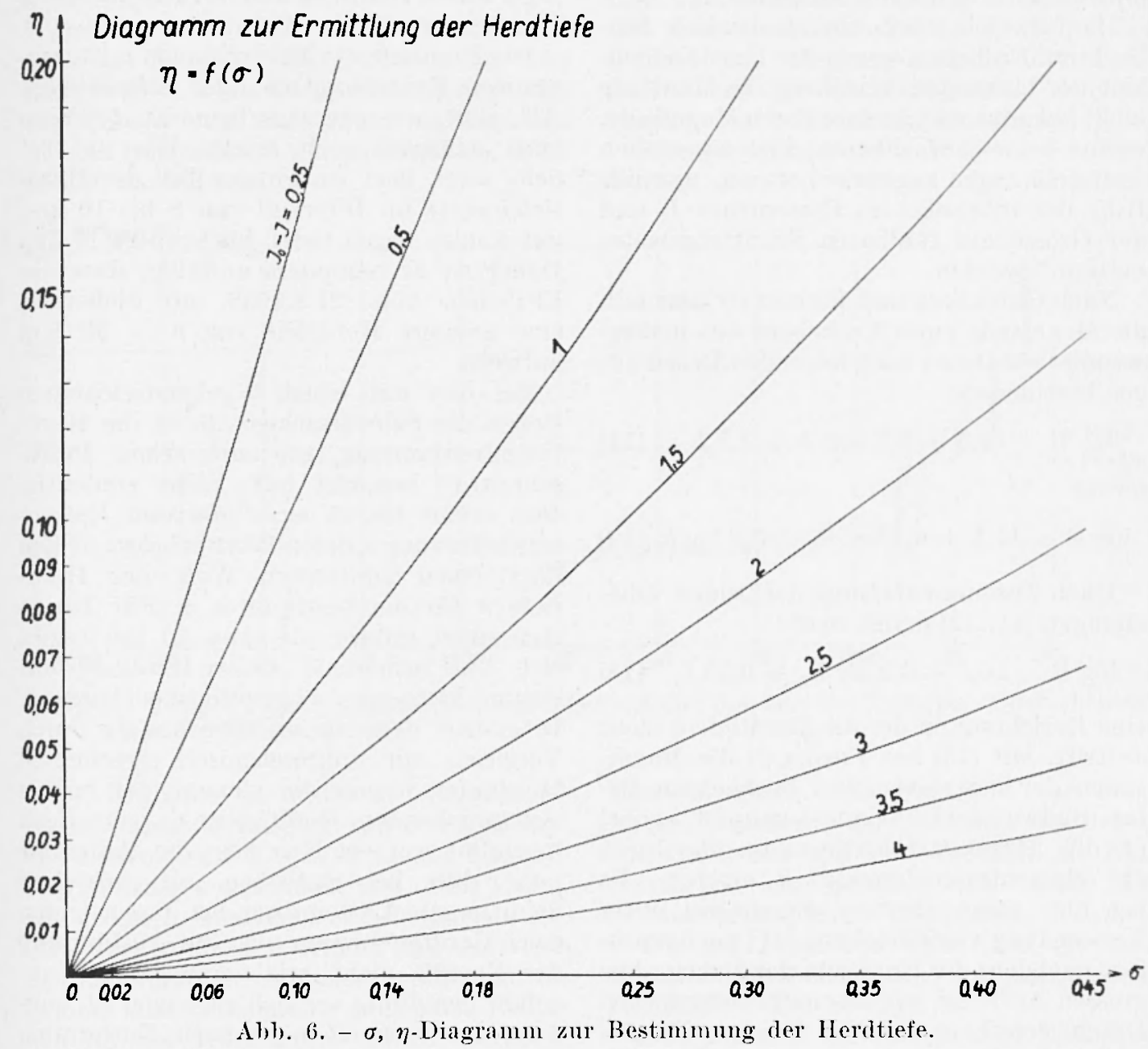

Hierzu liest man aus dem $\sigma, \eta$-Iiagramm (Abb. 6) die zugehörigen $\eta_{n}$-Werte $a b$, die durch a dividiert $h$ ergeben.

\begin{tabular}{ccccccc}
$\eta_{n}$ & 0,024 & 0,0225 & 0,025 & 0,024 & 0,026 & 0,024 \\
\hline$h_{n}$ & 24 & 22,5 & 25 & 24 & 26 & $24 \mathrm{~km}$
\end{tabular}

Abgesehen von der murermeidlichen Streuung sind die $h$-TVerte als konstant anzusehen, die Herdtiefe schwankt also in diesem Fall zwischen $h=22,5$ bis $26 \mathrm{~km}$. grossen Bereichs, so müssen wie schon erwähnt, die makroseismischen Daten als zu fehlerladt und daher als nicht geeignet für eine Herdtiefenberechnung verworfen werden. Dagegen würde die Anwendung eines der Rechenverfahren, etwa nach der Hethode der kleinsten Quadrate, in einem solchen Falle ein scheinbar gesichertes Ergebnis erbringen, dem in Wirklichkeit der physikalise he Simn fehlt.

Tach den bisherigen Erfahrungen mit der 
neuen Methode stimmen die hiermit errechneten Herdtiefen gut mit den mikroseismisch bestimmten uberein, während früher oftmals erhebliche Unstimmigkeiten $\mathrm{zwi}$ schen mikroseismisch und makroseismisch ermittelten Herdtiefen auftraten.

Da fur viele auch makroseismisch beobachtete Erdbeben wegen der Umständlichkeit der bisherigen Verfahren die Herdtiefe nicht bekannt war, konnte deren Magnitude, wenn keine brauchbaren instrumentellen Aufzeichnungen registriert waren, nur mit Hilfe der Intensität im Epizentrum $I_{0}$ und der Grösse des fuhlbaren Schüttergebietes betimmt werden.

Sach Gutenber'g und Richter $\left(^{3}\right)$ lässt sich die Magnitude eines Erdbebens aus makroseismischen Daten nach folgenden Bezielnungen bestimmen:

$$
\log \mathrm{E}=9,5+3,2 \log h+1,1 I_{o},
$$

fer'ner

$$
\log \mathrm{E}=11,1+6,+\log R_{h}-3,2 \log h
$$

Duch Zusammenfassung der beiden Gleichungen $[11,12]$ erhält man

$$
\log \mathrm{E}=10,3+3,2 \log \mathrm{R}_{h}+0,55 I_{o}
$$

eine Beziehung, in der die Herdtiefe $/$ nicht auftritt. Mit [13] hat Fiedler $\left({ }^{2}\right)$ die Magnituden der makroseismisch beobachten Beben Sudwestdeutschlands bestimmt, wobei er die Hrpozentralentfernung $R_{h}$ dureh die Epizentralentferumg $R$ ersetzte. Es lag nun nahe, Fiedlers Ergebnisse unter Verwendung von Gleichung [11] nachzuprüfen, nachdem die Herdtiefe der Beben ohne grossen Aufwand aus den makroseismischen Daten errechnet werden konnte. Hierbei stellte sich heraus, dass mit Ausnahme der in der Tabelle mit * bezeichneten Beben nur unbedeutende Unterschiede bei der Berechnung von log $\mathrm{E}$ nach [1.1] gegenüber der Bestimmung nach [13] auftraten. Allerdings muss hierzu bemerkt werden, dass nach der Definition ron Gutenber'g und Richter $R$ als der Radius des Schüttergebietes angesehen wird, das von der Isoseiste mit der Intensität $I=1,5^{\circ}$ eingeschlossen wird, während sich bei Fiedler in der Regel $R$ auf einen Schütterbezirkbezieht, der von der Isoseiste 3. Grades eingeschlossen wird. Die
Ubereinstimmung der Ergebnisse spricht für Fiedlers Auflassung von $R$. Ubrigens dürfte die Festlegung der Grenze des makroseismischen Bereichs im Sinne von Gutenberw und Richter heutzutage bei dem hohen Stonpegel durch Industrie und Verkehr nur noch in Ausnahmefällen möglich sein.

Die Herdtiefen in Deutschlands z. Zt. regsamstem Erdbebengebiet, der Schwübischen Alb, sind, wie eingangs bemerkt, teilweise auch mikroseismisch belegt. Wie die Tabelle zeigt, liegt ein grosser Teil der Herdtiefenwerte im Intervall von 8 bis $10 \mathrm{~km}$, nur wenige liegen tiefer bis etwa zu $15 \mathrm{~km}$. Daher ist es besonders auffällig, dass das Errlbeben rom 21.2.1933 mit Sicherheit eine grossere Herdtiefe ron $h=30 \mathrm{Km}$ aufweist.

Bei den mit einem * gekennzeichneten Beben der Schwäbischen Alb ist die Herrltiefenbestimmung, wie anch schon Peterschmitt $\left(^{6}\right)$ bemerkt hat, nicht eindentig. Man erhält bis zu einer gewissen Epizentralentfernung $s$, deren Wert bei etwa $50 \mathrm{Km}$ liegt, einen konstanten Wert der Herdtiefe $h$ für ein bestimmtes $\alpha$. Für Isosejstemradien grosser als etwa $50 \mathrm{~km}$ ergibt sich eine bedentend tiefere Herdlage mit einem kleineren Absorptionskoeffizienten. In einigen derartigen Fällen konnte durch Vergleich mit mikroseismisch errechneten Herdtiefen immer die kleinere der beiden sich ergebenden Herdtiefen als zutreffend bestimmt werden. Hier liegt die Anmahme nahe, dass bei Erdbeben mit grösserem Schuttergebiet, demn nur bei diesen treten zwei Herdtiefenwerte anf, die Ansbreitung der Energie nicht, wie rorausgesetzt, ungefähr geradlinig vor sich gegangen ist, sondern dass ron einer gewissen Entfermung an Energie zur Erdoberflache gelangt; die an einer tieferen Schicht entlang sich ausgebreitet hat. Erdbeben mit einer solchen Eigentümlichkeit fanden sich innerhalb Deutschlands bisher nur in der Schwäbischen Alb und in Oberschwaben. Hier zeigte sich bei dem Schadenbeben rom 27.6 . 1935 die vorerwähnte Eigentümlichkeit. In Ubereinstimmung mit den mikroseismischen Berechnungen wurde die Herdtiefe zu etwa $9 \mathrm{~km}$ ermittelt. Die Herdiefe des Bebens von 1898 ist dagegen weniger sicher, sie liegt zwischen 5 und $9 \mathrm{~km}$ Tiefe. 
Für die Erdbeben des Dornstetter Graben und des Albcorlandes wurde eine Herditiefe in der Grössenordnung ron etwa $15 \mathrm{~km}$ gefunden. In Gebiet südlich der Donan in Oberschwaben ergaben sich Herdtiefen ron 8 bis $9 \mathrm{~km}$.

Der Herd des Erdbebens rom 13.10. 1886 am Nordufer des Bodensees lay sehr fach in etwa $3 \mathrm{~km}$ Tiefe, während derjenige des Bebens rom 21.6. 1917 bei Konstanz die fur Deutschland schon erhebliche Tiefe von $\varrho 0 \mathrm{~km}$ aufweist.
Die drei Erdbeben im Niederheingebiet an Nordabfall der Eifel bzw. bei Aachen stimmen etwa mit ihren Herdtiefen, die bei $20 \mathrm{~km}$ liegen, überein. Ganz geringe Tiefen haben die Herde der Beben zu Herzogenlath und Tollhausen. Wenig unterscheiden sich auch die Herdtiefen der Erdbeben der Gegend ron Siegburg-Zülpich, der Erftgegend und der von Euskirchen, die einen Bereich ron 8 bis $12 \mathrm{~km}$ einnehmen.

Für das mitteldeutsche Erdbeben mit dem Epizentrum am Ostrand des Thüringer

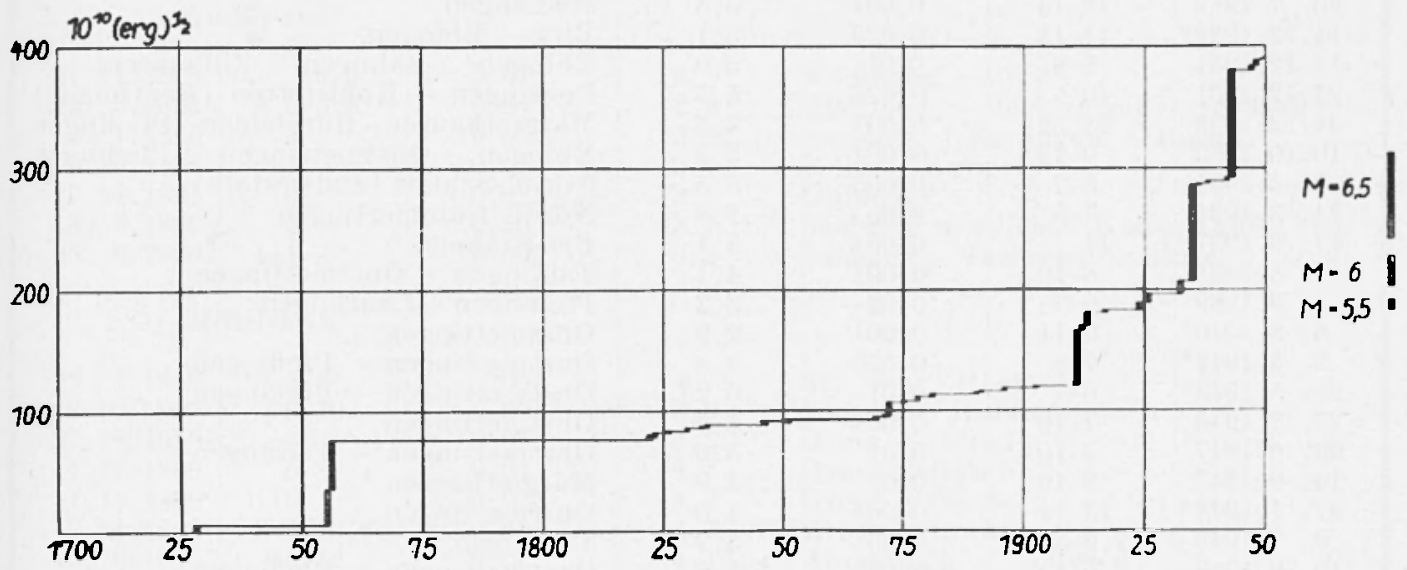

Abb. 7. - Preigewordene Spannungsenergie als Funktion der Zeit für Erdbeben Dentselılands mit einer Magnitude $M \geqq 3,3$.

Die Erdbeben der mitleren schuerzwaldregion haben Herdtiefen ron 8 bis $13 \mathrm{~km}$, das Beben rom 3.8.1728 in der Gegendron Kenzingen eine solche ron $1+$ bis $17 \mathrm{~km}$. Die grossten Herdtiefen auf deutschem Boden wurden in der Gegend des südlichen Schwarzwaldes erreicht, hier wurden für die beiden kurz aufeinander folgenden istbeben an der Hornisgrinde $26 \mathrm{~km}$ Herdtiefe nachgewiesen.

Die Erdbeben in der Rheinebene liegen mit Ausnahme des Erdbebens rom 24 . 1 . 1880 bei Langenkandel in ganz geringen Tiefen ron 3 bis $6 \mathrm{~km}$. Von derselben Grössenordnung sind sie Herdtiefen der Erdbeben in der Gegend des Kaiserstuhls, ciner Vulkanruine, wenn man das Erdboben rom 29.6. 1926 bei Sasbach-Endingen-Riegel ausnimmit.

Die Herde der Erdbeben in Gebiet des Mittelrheins ron Koblenz bis Grossgerau liegeen in einem Tiefenbereich ron 6 bis $12 \mathrm{~km}$.
Beckens wurde eine Herdtiefe ron etwa $15 \mathrm{~km}$ bestimmt, dagegen sind die Beben ron Stadtroda und rom Werratal als Einsturzbeben anzüsprechen und haben daher nur sehr geringe Hereltiofen.

Für das Eragebirge stand nmr ein answertbares Erdbeben zur Verfügung, dessen Herdtiefe $8 \mathrm{~km}$ beträgt.

Ferner sind in der Tabelle zwoi Erdbeben in Schlesien mit Herdtiefen ron etwa $9 \mathrm{~km}$ angreführt.

Aus den zusammengestellten Ergebnissen scheint hervorzugehen, dass abgesehen ron wenigen Ausnalimen jerles Herdgebiet in Deutschland eine gewisse ihm eigentümliche Herdtiefe aufweist. Die grösste bisher nachgewiesene Tiefe liegt bei $30 \mathrm{~km}$.

Grössere Schwankungen, auch innerhalb eines eng begrenzten Herdgebietes, zeigt der Absorptionskoeffizient $\alpha$. Beispielsweise liegen die Werte dieser Grösse in Bereich der Schwäbischen Alb zwischen $\alpha=0,001$ und 
T a belle

\begin{tabular}{|c|c|c|c|c|}
\hline Datum & $\begin{array}{c}\text { Herltiefe } \\
h \\
[k m]]\end{array}$ & $\begin{array}{c}\text { Alsorptions } \\
\text { hoellizient } \\
\alpha \\
{[\mathrm{km}]^{-1}}\end{array}$ & $\begin{array}{c}\text { Magnituile } \\
\ldots\end{array}$ & Epizentrum \\
\hline \multicolumn{5}{|c|}{ 1) Sciuabische Alb } \\
\hline $\begin{array}{r}23.9 .1830 \\
24.11 .1872 \\
13.7 .1875 \\
16.11 .1911 * \\
20.7 .1913 \\
11.12 .1924^{*} \\
11.12 .1931 \\
22.12 .1931 \\
21.2 .1933 \\
10.10 .1933 \\
1.1 .1934 \\
24.3 .1934 \\
17.61 .1937 \\
2.8 .1938 \\
1.3 .1939 \\
6.8 .1940^{*} \\
2.5 .1943^{*} \\
28.5 .1943^{*} \\
27.12 .1943 \\
28.6 .1947 \\
14.99 .1947 \\
27.1 .1948^{*} \\
8.7 .1949 \\
15.9 .1949 \\
6.11 .1949\end{array}$ & $\begin{array}{c}12 \\
10,5-11 \\
11-12 \\
13-17 \\
12-14 \\
14-15 \\
8-9 \\
10 \\
28-32 \\
9-12 \\
5-7 \\
7-8 \\
11 \\
8-10 \\
6-7 \\
9-11 \\
7-8 \\
6-7 \\
7-10 \\
9-10 \\
8-10 \\
13-14 \\
7,5-8 \\
5 \\
7-8\end{array}$ & $\begin{array}{l}0,(025 \\
0,01 \\
0,01 \\
0,001 \\
0,001 \\
0,025 \\
0,01 \\
0,025 \\
0,001 \\
0,001 \\
0,0(0) 5 \\
0,05 \\
0,001 \\
0,001 \\
0,02 \\
0,001 \\
0,025 \\
0,01 \\
0,001 \\
0,01 \\
0,01 \\
0,001 \\
0,05 \\
0,025 \\
0,01\end{array}$ & $\begin{array}{l}4,5 \\
3,2 \\
5,3 \\
6,3 \\
5,5 \\
5,1 \\
5,0 \\
5,5 \\
4,5 \\
3,3 \\
5,5 \\
2,8 \\
3,1 \\
4,1 \\
3,3 \\
2,9 \\
5,8 \\
6,23 \\
5,0 \\
5,4 \\
2,9 \\
4,5 \\
3,2 \\
2,8 \\
4\end{array}$ & 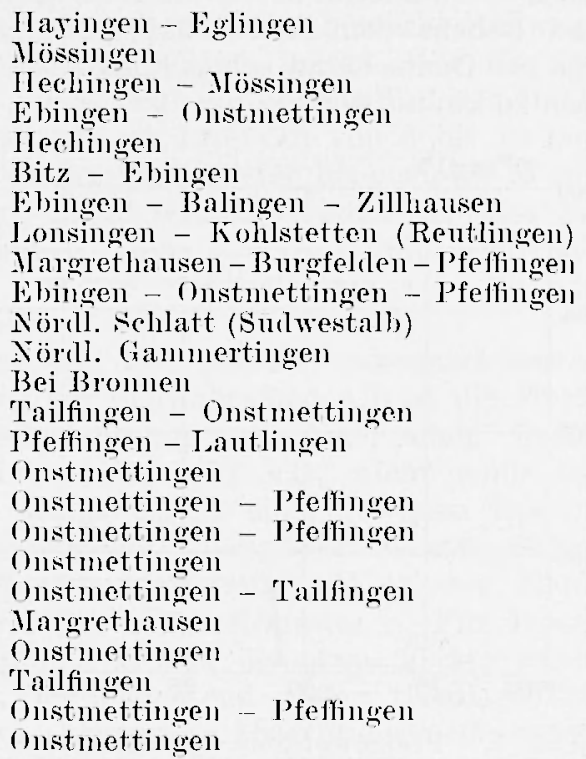 \\
\hline \multicolumn{5}{|c|}{ 2) Oberschuaben } \\
\hline $\begin{array}{r}\text { 6. 10.1898 } \\
27.0 .1935^{*} \\
\text { 3) } 10_{07}\end{array}$ & $\begin{array}{c}\qquad-5 \\
8,5-9,5 \\
\text { nsleller Gral }\end{array}$ & $\begin{array}{c}0,001 \\
0,025 \\
e n-.1 l b \text { corla }\end{array}$ & $\begin{array}{l}3,9 \\
6,0\end{array}$ & $\begin{array}{l}\text { Saulgau - Mengen } \\
\text { Saulgau - Herbertingen }\end{array}$ \\
\hline $\begin{array}{l}28.11 .1822 \\
19.4 .1936\end{array}$ & $\begin{array}{l}13-16 \\
14\end{array}$ & $\begin{array}{l}0,025 \\
0,01\end{array}$ & $\begin{array}{l}5,1 \\
4,5\end{array}$ & $\begin{array}{l}\text { Yrurgtal (Dornstetter Graben) } \\
\text { Albyorland bei Tübingen, Bebenhausen } \\
\text { - IIagerloch }\end{array}$ \\
\hline \multicolumn{5}{|c|}{ 4) Bodensee } \\
\hline $\begin{array}{l}13.10 .1886 \\
21.6 .1917\end{array}$ & $\begin{aligned} 4 \\
20\end{aligned}$ & $\begin{array}{l}0,01 \\
0,001\end{array}$ & $\begin{array}{l}2,74 \\
+, 5\end{array}$ & $\begin{array}{l}\text { Bei Friedrichshafen, Bodensee Nordufer } \\
\text { Konstanz, westlicher Bodlensee }\end{array}$ \\
\hline \multicolumn{5}{|c|}{ 5) Snhuarzuald } \\
\hline $\begin{aligned} & \text { 3. } 8.1728 \\
& 24 . 1.1883 \\
& 21 . 4.1885 \\
& 7 . 0.18865 \\
& 13 . 1.1895 \\
& 22 . 1.1896 \\
& 30.12 .1935\end{aligned}$ & $\begin{array}{l}14-17 \\
10-13 \\
11-12 \\
8 \\
8,5-10,7 \\
13 \\
26\end{array}$ & $\begin{array}{c}0,001 \\
0,01 \\
0,001 \\
0,01-0,001 \\
0,01 \\
0,005 \\
0,001\end{array}$ & $\begin{array}{l}5,3 \\
3,4 \\
3,1 \\
2,6 \\
3,5 \\
4,8 \\
5,1\end{array}$ & $\begin{array}{l}\text { Kenzingen - Lahrer Bruch } \\
\text { Gottenheim (südlicher Schwarzwald) } \\
\text { Feldberg. } \\
\text { Hittlerer Schwarzwald bei Jahr } \\
\text { Bonndorfer Graben } \\
\text { Neustalt - Kappel - Saig } \\
\text { Hornisgrinde }\end{array}$ \\
\hline
\end{tabular}




\begin{tabular}{|c|c|c|c|c|}
\hline Datum & $\begin{array}{c}\text { Ilerdtiefe } \\
h \\
{[k m]}\end{array}$ & $\left|\begin{array}{c}\text { Absorptions. } \\
\text { koeffizient } \\
\alpha \\
{[\mathrm{kml}]^{-1}}\end{array}\right|$ & $\begin{array}{l}\text { Magnitude } \\
\qquad I\end{array}$ & Epizentrum \\
\hline \multicolumn{5}{|c|}{ 6) Oberrheintalgraben } \\
\hline $\begin{aligned} 7 . & 2.1839 \\
24 . & 1.1880 \\
11 . & 6.1887 \\
8 . & 2.1933 \\
7 . & 6.1948\end{aligned}$ & $\begin{array}{r}8-10 \\
8-9 \\
3,5 \\
4-6,5 \\
5-6,5\end{array}$ & $\begin{array}{l}0,05 \\
0,005 \\
0,04 \\
0,07 \\
0,001\end{array}$ & $\begin{array}{l}4,9 \\
3,4 \\
3,2 \\
5,4 \\
5,1\end{array}$ & $\begin{array}{l}\text { Zaber Enz- Wurmgebiet } \\
\text { Langenkandel } \\
\text { Schuttern bei Lalur } \\
\text { Rastatt } \\
\text { Karlsruhe }\end{array}$ \\
\hline \multicolumn{5}{|c|}{ 7) Kaiserstuhl } \\
\hline $\begin{array}{r}21.11 .1823 \\
24.6 .1884 \\
3.1 .1886 \\
9.10 .1886 \\
17.11 .1891 \\
14.2 .1899 \\
28.6 .1926\end{array}$ & $\begin{array}{l}6,5 \\
+, 5-5,5 \\
3 \\
3 \\
5 \\
5 \\
11\end{array}$ & $\begin{array}{l}0,07 \\
0,04 \\
0,05 \\
0,05 \\
0,025 \\
0,25 \\
0,005\end{array}$ & $\begin{array}{l}+ \\
2,9 \\
2,52 \\
3,6 \\
3 \\
3,7 \\
5,5\end{array}$ & $\begin{array}{l}\text { Endingen } \\
\text { Endingen - Jechtingen - Sashach } \\
\text { Sashach - Endingen - Riegel } \\
\text { Kehlu- Kappel } \\
\text { Opfingen } \\
\text { Sashach westlicher Kaiserstuhl } \\
\text { Sasbach - Inringen - Riegel }\end{array}$ \\
\hline \multicolumn{5}{|c|}{ 8) Willelrhein } \\
\hline $\begin{array}{r}29.7 .1846 \\
2 . / 3.10 .1869 \\
31.10 .1869 \\
1.11 .1869 \\
1.11 .1869 \\
2.11 .1869 \\
19.6 .1928\end{array}$ & $\begin{array}{l}6,5-8 \\
8-10 \\
6 \\
6-8 \\
10 \\
8,6-10,6 \\
10-12\end{array}$ & $\begin{array}{l}0,001 \\
0,001 \\
0,05 \\
0,04 \\
0,025 \\
0,05 \\
0,07\end{array}$ & $\begin{array}{l}4,7 \\
3,9 \\
4,3 \\
4,4 \\
4,1 \\
4,3 \\
4,13\end{array}$ & $\begin{array}{l}\text { St. Goar, Rheintal } \\
\text { Engers - Koblenz } \\
\text { Gross - Gerau } \\
\text { Gross - Geraul } \\
\text { Gross - Geraul } \\
\text { Gross - Gerau } \\
\text { Andernach - Neuwied }\end{array}$ \\
\hline \multicolumn{5}{|c|}{ 9) Niederhein. Aachener Gebied } \\
\hline $\begin{array}{r}26.27 .12 .1755 \\
18.2 .1756 \\
22.10 .1873 \\
24.6 .1877 \\
26.8 .1878 \\
18.11 .1881 \\
13.12 .1928 \\
11.7 .1949 \\
8.3 .1950 \\
14.3 .1951\end{array}$ & $\begin{array}{c}18-21,5 \\
21-25 \\
3,7-4,5 \\
2 \\
4-5 \\
22-24 \\
10,5-11,5 \\
12 \\
6,5-8,5 \\
9\end{array}$ & $\begin{array}{l}0,0012 \\
0,006 \\
0,04 \\
0,01 \\
0,001 \\
0,01 \\
0,02 \\
0,02 \\
0,01 \\
0,0(010\end{array}$ & $\begin{array}{l}5,8 \\
6,2 \\
4,2 \\
4,05 \\
5,05 \\
4,7 \\
4,1 \\
4,25 \\
4,4 \\
5,2\end{array}$ & 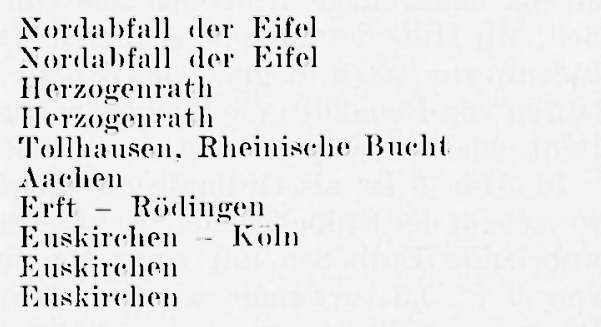 \\
\hline \multicolumn{5}{|c|}{ I0) Milleldeulschland } \\
\hline $\begin{array}{ll}\text { (j. } & 3.1872 \\
28 . & 1.1927 \\
22 . & 2.1953\end{array}$ & $\begin{array}{l}14, \tilde{5}-16,5 \\
\quad 3 \\
1,1\end{array}$ & $\begin{array}{l}0,001 \\
0,01 \\
0,04\end{array}$ & $\begin{array}{l}5,6 \\
3,14 \\
3,57\end{array}$ & $\begin{array}{l}\text { Ostrand des Tüuringer Beckens, Ron- } \\
\text { neburge } \\
\text { Stadtroda (Thü̈r. Becken, östlicher Tel) } \\
\text { Werratal }\end{array}$ \\
\hline \multicolumn{5}{|c|}{ 11) Vogll. Eraebirge } \\
\hline \multicolumn{4}{|c|}{ 12) Schlesien } & Plamen \\
\hline $\begin{array}{ll}\text { 31. } & 1.1883 \\
11 . & 6.1895\end{array}$ & $9-10$ & $\begin{array}{l}0,001 \\
0.001\end{array}$ & $\begin{array}{l}3,67 \\
3,97\end{array}$ & $\begin{array}{l}\text { Trautenau } \\
\text { Mittel - Schlesien: strehlen, Reichen- } \\
\text { hacll }\end{array}$ \\
\hline
\end{tabular}


$a=0,0 \%$. Nach den vorliegenden Erfahrungen ist $a=0,001$ als muterer Grenzwert anzusehen. Die obere Gienze ist rlagegen nicht ganz sicher. Im Rahmen dieser Untersuchung wurlen die hörhsten Werte für den Absorptionskoeffizienten in Oberrheintalgraben gefunden, anscheinemd bedingt durch rlie hier vorhandene grosse Mächtigkoit der lockeren Serlimente. Wrerte ron $a=0,07$, in Falle des Bebens rom 14.2. 1899 sogar $\alpha=0,25$, wurdell hier erreicht. Selbst wenn man diesen letzteren Wert als unsichere, etwa durch besonderes Umstande volgetäuscht, ansieht, so bleibt: dennoch für den Absorptionskocffizienten cin Schwankungsbereich von ungefähr $a=$ 0,001 bis fast 0,1. Es muss allerdings hierbed berïcksichtigt werden, dass der $\Delta$ bsorptionskoeffizient keine einfarche physikalische Grösse, wie z B. die Ausbreitungsgeschwindigkeit elastischer Wellen, darstellt, sondern dass er ron melneren Faktoren abhängt. Abgesehen von den elastischen Konstanten des Mediums wirken hier noch die Frequenz der Borlensehwingung und die Art der Wellenausbreitung mit, die entweder räumlich orler innerhalb einer Schicht vor sich gehen katun.

Die Tabelle enthält die wichtigsten Errbeben vom Jahre 1800 an sowie darüber hinaus noch einige Erolbeben aus früherer Zeit. Mit Hilfe der dazn berechneten Magnitudenwerte ist es möglich, nach dem Terfahreen ron Benioff (1) die Errbebentätigkeit Deutschlands energiemässig datyustellen.

In Abb. 6 ist als Ordinate die Quadratwurzel aus der Erdbebenenergie aufget ragen, wobei alle Errbeben mit einer Magniturle ron $I I \geqq 3,3$ verwendet wurken. Aus dem Diagramm geht hervor, dass nach der Jahrhundertwende eine bedentende Zmohme der Seismizität in Deutschland zu verzeichnem ist. Jerloch ist riesere Zuwarchs grösstenteils auf die Erdbebentätigkeit rler Schwäbischen Alb seit 1911 zurückzufuhren.

\section{ZUSA M MENFASSUNG}

Eine neue maliroseismische Methode gestattet die schnelle und sichere Ermittlung der Herditiefe fiur jeden mittleven Isoseistenabstand vom Elpizentrum. Hierbei wird ein Diagramm benutzt, dem die Gleichung von
Kövesligethy zugrunde liegt. Die auf diese Weise erhaltenen Werte der Herdtiefen derjenigen Erdbeben Deutschlands, von denen Isoseistenlarten yezeichnet werden liomiten, zeigen eine gerisse Cbereinstimmung für jedes Epizentralyebiet. Die aus Epizentralintensitrit und Radius des schüttergebietes bzu. aus Epizentralintensitat und Herdtiefe erechneten Magnitudenuerte stimmen im allgemeinen uberein. Die Darstellung der ausgelösten spamungen in Abhängiglieit ion der Zeit fiir alle Erdbeben Deutschlands mit einer Hagnitude ron $M \geqq 3,33$ zeigt ein starles scheinbares Ansteigen der Errbebentatiglieit seit 1911 .

\section{ABSTRACT}

A new macroseismic method permits the quick and sure determination of the focal depth for each middle isoseismal distance from epicenter. Herewith a diagram is used, which is founded on Kövestigethy's equation. The focal depth of earthuulies in Germany. determined in this mamner, to which isoscismal maps could be draten, showes a certain accord for each epicentral region. The magnitudes computed from epicentral intensity and radius of proceptibility or from epicentral intensity and focal depth accord generally. The representation of strain release in function of time for all earthqualies of Germany with $M \geq 3,3$ shows an important apparent ascent since 1.911.

\section{RIASSTNTO}

In muovo metorlo macrosismico permette la determinazione rapida e sicura della profondità ipocentrale per ogni distanza isosismica media dall'epicentro.

A quest'uopo ci si serve di un diagramma ricavato dalla formula del Köovesligethy.

I valori delle profondita dei focolari ottenuti con questo metorlo per quei terremoti della Germania, di cui si potevano disegnare delle carte isosismiche, mostrano una certa conformità per ogni territorio epicentrale.

I valori di magnitudo calcolati rispettivamente dall'intensità epicentrale ed il raggio del territorio di scossa oppure dall'intensità 
apiecntrale e la profonditì ipocentrale corrispondono generalmente.

Il grafico delle tensioni stincolate in dipendenza dal tempo, per tutti $i$ terremoti della Germania con una magnitudo di $M \geq 3,33$ mostra un'apparente jorte crescendo dell'attivitì sismica dal 1911.

\section{LITTERATI*R}

(1) Bexiofr, II., Gilobal strain and Release as Revealed by Great Earlhqualies. " (Beol. Soc Amer." 62 . (1951).

$\left(^{2}\right)$ FEDLER, G., Die Erdbebentatiglieit in. Sïdwestdeutschland 1800-19:0\%. Dissertation. stuttgart, 1954.
(1) Getesberg, B. und Richter, ('. F.. Eam quate Magnitude Inlensitu, Energy and Acceleration. "Bull. Seism. Soc. Amer." 23, 3( . Juli, 1943).

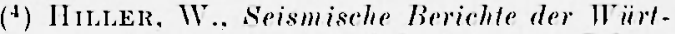
tembergischen Embebenuarten, Jahrg. 1935. Stuttgart, 1936.

(5) Körestagetur, v. R.. Seismischer Starlie. arad und Intensitat der Beben. "Gerlands Beitrige zur fieophrsik o. Bd. VIll. leiprig. $190 \%$.

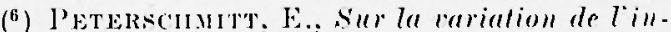
tensite macroséismique aree la distance epicentrale. " P'ubl. Burean Central séismol. Intern. "Serie $\mathrm{A}$. Is (1952).

(') Sposneusk, W., Methoden zur IIerdtiefenbestimmung in aer Maliroseismil (.1ls Manuskipt gedruckt.) 1958. 